\title{
Revisiting broadband reflection suppression by Mie scatterers: the role of electromagnetic duality
}

\author{
Evgenila Slivina ${ }^{*}, 1,2$, Aimi Abass ${ }^{2}$, Derk Bätzner ${ }^{1}$, Carsten \\ Rockstuhl $^{2,3}$, And IVAn Fernandez-CORBATON ${ }^{2}$ \\ ${ }^{1}$ Meyer Burger Research AG, Rouges-Terres 61, 2068, Hauterive, Switzerland \\ ${ }^{2}$ Karlsruhe Institute of Technology, Institute of Nanotechnology, \\ Hermann-von-Helmholtz-Platz 1, 76344, Eggenstein-Leopoldshafen, Germany \\ ${ }^{3}$ Karlsruhe Institute of Technology, Institute of Theoretical Solid State Physics, \\ Wolfgang-Gaede-Str. 1, 76131, Karlsruhe, Germany \\ Evgeniia.Slivina@meyerburger.com
}

\begin{abstract}
The physics behind broadband reflection suppression that high index dielectric nanoparticle arrays can provide is revisited. We demonstrate that antireflective properties are inherently connected to how equal the effective magnetic and electric responses are.
\end{abstract}

OCIS codes: 000.0000, 240.0240, 350.6050.

This is a draft manuscript of a paper published in Light, Energy and the Environment 2018 (E2, FTS, HISE, SOLAR, SSL), OSA Technical Digest (Optical Society of America, 2018).

The full citation is: E. Slivina, A. Abass, D. Bätzner, C. Rockstuhl, and I. Fernandez-Corbaton, "Revisiting broadband reflection suppression by Mie scatterers: the role of electromagnetic duality", in Light, Energy and the Environment 2018 (E2, FTS, HISE, SOLAR, SSL), OSA Technical Digest (Optical Society of America, 2018), paper JM4A.3.

The digital object identifier (DOI) is: 10.1364/EE.2018.JM4A.3. One print or electronic copy may be made for personal use only. Systematic reproduction and distribution, duplication of any material in this publication for a fee or for commercial purposes, or modification of the contents of the publication are prohibited.

\section{Introduction}

Decorating silicon wafer with high-index nanoparticle arrays demonstrated a superb anti-reflection (AR) performance, surpassing that of chemically textured standard wafer. However, the direct structuring leads to an increase in surface recombination and thus makes passivation of the cell challenging. It 
has been furthermore shown that the usage of $\mathrm{TiO}_{2}$ Mie scatterer array may accomplish a similar effect while avoiding surface damage though with worse anti-reflection performance. The physical mechanism behind how these high index scatterers suppress reflection, however, is not well understood, with most attributing it to the absorber material's higher optical density of states by virtue of higher refractive index [1].

Here, we demonstrate that the anti-reflection property of such arrays is inherently connected to how close the nanoparticle array brings the system to the duality condition where the effective magnetic and electric responses are equal. This physical insight reveals another symmetry requirement that needs to be fulfilled by the nanoparticle array to guarantee excellent antireflection performance, namely possessing at least $C_{3}$ rotational symmetry or higher $\left(C_{4}\right.$, etc.). To show the relation between anti-reflection performance and duality, we consider the optimization problem of an electrically decoupled light trapping structure comprising of a $\mathrm{TiO}_{2}$ nanodiscs array placed at the front side of an HJT solar cell. When the geometry of the nanodiscs and the array arrangement are properly tailored, reflectance can be suppressed to below $5 \%$ over a broad spectral region relevant for c-Si based solar cells. We show that spectral regimes corresponding to high reflection in our optimized solar cell structure are essentially regimes where one is significantly far from the duality condition for the entire sample. Based on this physical insight and the rotational symmetry requirement, design guidelines on the particle size, shape and arrangement may be inferred.

\section{Results and discussion}

A system that has a discrete rotational symmetry of order 3 or higher, and that also has electromagnetic duality symmetry will necessarily exhibit zero backscattering [2]. This result is a generalization of the well-known first Kerker condition: a sphere with equal permeability and permittivity exhibits zero backscattering [3]. The $\epsilon_{r}=\mu_{r}$ condition means perfect duality symmetry independently of the geometry. In the particular case of a sphere it also means that the electric Mie coefficients are equal to the magnetic Mie coefficients for all multipolar orders. Shape optimized dielectric objects with $\mu_{r}=1$ can approximate the duality symmetry by the overlap of their electric and magnetic multipolar responses. This effect can be further extended to a case of a nanoscatterer placed on top of a substrate. This substrate-mediated Kerker effect is the result of the destructive interference of EM field reflected from the surface of a substrate with the fields associated with radiating electric and magnetic multipoles excited in the nanoparticle. Suppression of reflection in a broadband manner was shown for silicon nanosphere arrays placed on top of a substrate [4]. However, such backscattering suppression is possible not only for spherically shaped particles, but could be achieved for scatterers of various shapes as long as rotational symmetry condition is met. For instance, cylindrical particles are superior to spheres since they 
(a)

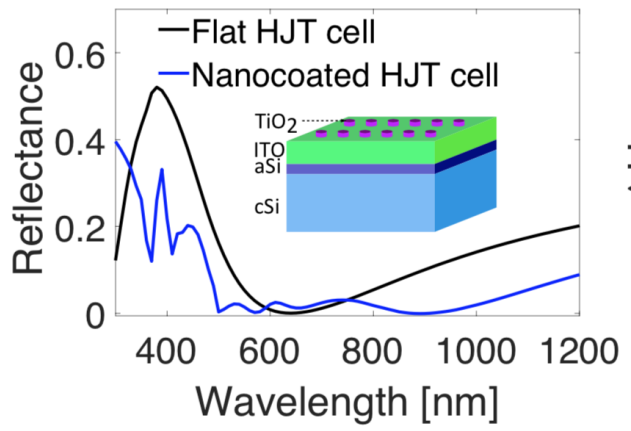

(b)

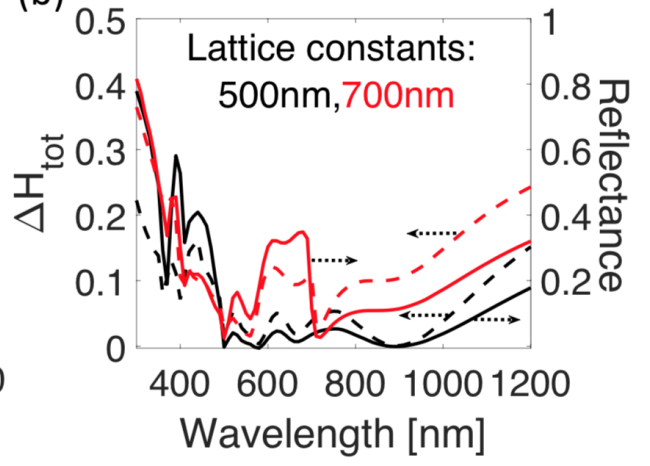

Figure 1: (a) Reflectance from a $\mathrm{HJT}$ cell coated with square $\mathrm{TiO}_{2}$ nanoparticle array. (b) Figure of merit of helicity change corresponding to the total field power (Equation 1) vs reflectance. Incoming field polarization is always left handed.

possess an additional geometrical degree of freedom which leads to a spectral overlap of the electric and magnetic dipole resonances. Here, we consider a square array $\left(C_{4}\right.$ symmetry) of low-absorptive $\mathrm{TiO}_{2}$ Mie scatterers on the front surface of a heterojunction solar cell (inset of Figure 1(a)). This nanodisc array allows for a significant improvement in AR performance over the broad wavelength range, when tuned accordingly Figure 1(a).

The spectral range, which exhibits low reflections, is in fact the range where the system $\left(\mathrm{TiO}_{2}\right.$ array and layer stack) approaches the electromagnetic duality condition. For dual structures, scattering does not result in a change of helicity of light [2]. Therefore, the analysis of how much light is coupled into the helicity opposite to that as used for the illumination, allows to conclude on how dual the system is. Helicity can be understood while considering electromagnetic field decomposition into plane waves. In this case, preservation of helicity can be defined as preservation of the electric field handedness for circularly polarized incident light. In the following we probe the response of the entire system upon illuminating it with a plane wave of specific handedness and study the fraction of light that is scattered into the opposite handedness. To quantify this change in helicity we consider as a measure the following expression:

$$
\Delta H_{\text {tot }}=P_{\text {scat }, \text { right }} /\left(P_{\text {scat }, \text { left }}+P_{\text {scat }, \text { right }}+1\right),
$$

where $P_{\text {scat,left/right }}$ corresponds to backscattered power. The powers that appear in this expression are normalized to the incident power. It can be seen in Figure 1(b) that, even though at shorter wavelengths we do encounter higher diffraction orders, the measure defined above is an excellent proxy for the reflectance and both quantities have the same functional dependency. Indeed, reflectance comes hand-in-hand with the helicity change. 


\section{Conclusion}

We show that suppression of reflection in a broadband manner is closely linked to how well the system approaches the electromagnetic duality condition. To reach this modi operandi in an optimal sense we require to induce in the scatterer (that consists here of the combined system of an actual structure on top of the layer stack) simultaneously electric and magnetic dipole moments of equal strength across a large spectral region. Based on this physical insight, design guidelines on the particle size, shape and arrangement may be made that can efficiently be used in solar cells.

\section{References}

[1] P. Spinelli, M. A. Verschuuren, and A. Polman, Broadband omnidirectional antireflection coating based on subwavelength surface Mie resonators, Nat. Commun. 3, 692 (2012).

[2] I. Fernandez-Corbaton, Forward and backward helicity scattering coefficients for systems with discrete rotational symmetry, Optics Express 21, 29886 (2013).

[3] M. Kerker, D.-S. Wang, and C. L. Giles, Electromagnetic scattering by magnetic spheres, J. Opt. Soc. Am. 73, 765-767 (1983).

[4] K. V. Baryshnikova et al., Plasmonic and silicon spherical nanoparticle antireflective coatings, Scientific Reports 6, 22136 (2016). 